\title{
Measurement of Visual and Auditory Stimuli Using EEG Headset Emotiv Epoc+
}

\author{
Martina Zabcikova ${ }^{1, *}$ \\ ${ }^{1}$ TBU in Zlin, Faculty of Applied Informatics, Department of Informatics and Artificial Intelligence, 76005 Zlin, Czech Republic
}

\begin{abstract}
Brain-Computer Interface (BCI) has received a huge interest as a direct communication pathway between a human brain and an external device. BCI is very useful in many areas of research. This study examines and discusses the feasibility and usability of the Emotiv Epoc+ noninvasive device. The focus is on the analysis of electroencephalography (EEG) signals associated with visual and auditory senses. To measure signals the free version of software Emotiv Xavier ControlPanel is used. The results depict that the Emotiv Epoc+ device is a suitable option in BCI for scientific and entertainment purposes.
\end{abstract}

\section{Introduction}

Brain-Computer Interface (BCI) creates a direct communication between the brain and an external device. Real-time interaction with the brain using BCI technologies is opening new, important, and potentially highly impactful possibilities for improving the lives of people. BCI research has shown enormous potential in a wide range of applications. Possible examples for the application of BCI devices include the restoration of communication function for people suffering ALS, treatment of movement disorders, detection and suppression of epileptic seizures, rehabilitation of chronic stroke survivors, or treatment of people with severe chronic pain. The substantial improvement of BCI research is documented by the large and growing academic researchers in this area. Research and studies provided ample evidence that signals recorded invasively or noninvasively from the brain can be used to give information about the motor, sensory, cognitive, or affective state of a user. That information can be decoded from the brain in real time, and the results of this decoding can produce stimulation of the nervous system (to replace, restore, enhance, supplement, or improve human functions). [1]

BCI device is used especially to help disabled people. However, these research approaches also aim at applications that are not necessarily in the clinical field. Many BCI applications currently exist. Emerging areas are medicine, communication and control, rehabilitation, assistive technology, entertainment, training, security, psychology, etc. [2]

BCI systems consist of several sequential steps, which can be categorized into four groups. It is about a brain activity pattern generation, signal acquisition, feature extraction, and classification. The appropriate technique should be chosen according to the type of brain activity and the purpose of the measurement. There are many types of BCI. The most common is the division into invasive and noninvasive systems. Another way to categorize BCIs is through their Operation Strategy, in which the BCI elicits brain signals during use. The BCI that utilizes selective attention presents users with auditory, visual, or tactile stimulation to elicit brain signal responses. [3] Many classes of electrophysiological activities of the brain are used in designing various types of BCIs. Sensorimotor activity corresponds to the behavior of the brain rhythms (mu, beta, and gamma), movement-related potentials and other. [4]

The usability and feasibility are an indispensable part of BCI systems. In this article, brain signals were measured using Emotiv Epoc+ device. Emotiv is one of the BCI technology for public users. To measure brain activity using Emotiv devices noninvasive BCIs based on EEG recordings is used. In which the electrodes are placed on the surface of the skull. To examine brain activity, the record of spontaneous brain activity of visual and auditory stimuli is used. Various electrodes in the Emotiv Epoc + mobile wireless neuroheadset provide the data. To measure signal quality is used Emotiv Xavier ControlPanel software.

\section{Emotiv Epoc+}

Emotiv Epoc+ is designed for scalable and contextual human brain research. Provides access to professional grade brain data with a quick and easy to use design. Uses fourteen channel EEG that senses whole brain activity. Saline-based electrodes are with wet sensors. Minimal

${ }^{*}$ Corresponding author: zabcikova@utb.cz 
setup time is only about five minutes. This device works wirelessly. You may connect your computer or mobile phone. This device lasts for up to twelve hours. To detect head movements, nine-axis motion sensors are available. [5]

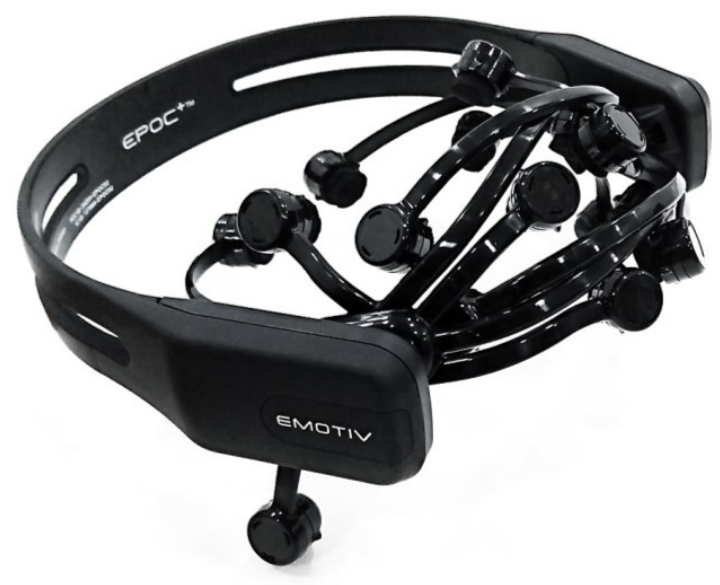

Fig. 1 Headset Emotiv Epoc+. [5]

There are fourteen EEG sensors for sense brain activity and two CMS/DRL references (left/right mastoid process alternative). CMS (Common Mode Sense) is an active electrode and DRL (Driven Right Leg) is a passive electrode. [5]

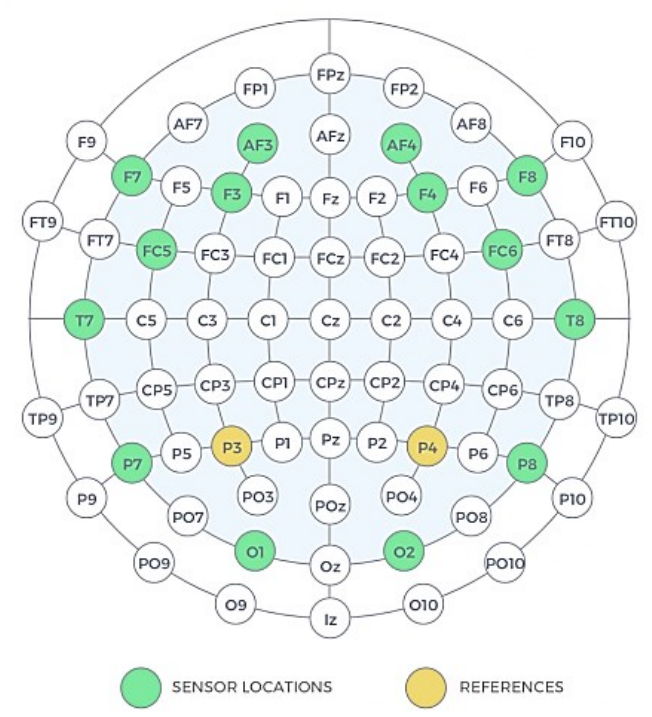

Fig. 2 Locations of sixteen EEG sensors. [5]

The yellow sensors are the basic electrode connected to the reference electrode. They are unipolar and located on the ears and thereby acquire basic input data. Green sensors are located in the frontal, temporal, parietal and occipital part of the head. Green sensors show a bipolar connection that is virtually obtained by subtracting the values of two adjacent electrodes. The advantage of this process is the elimination of amplitude artifacts. [5]

\section{Emotiv Xavier ControlPanel}

The measurement of the signal is being done with free software Emotiv Xavier ControlPanel. Available detections in this app are mental commands, performance metrics, and facial expressions. Here are used performance metrics that include excitement, engagement, relaxation, interest, stress, and focus.

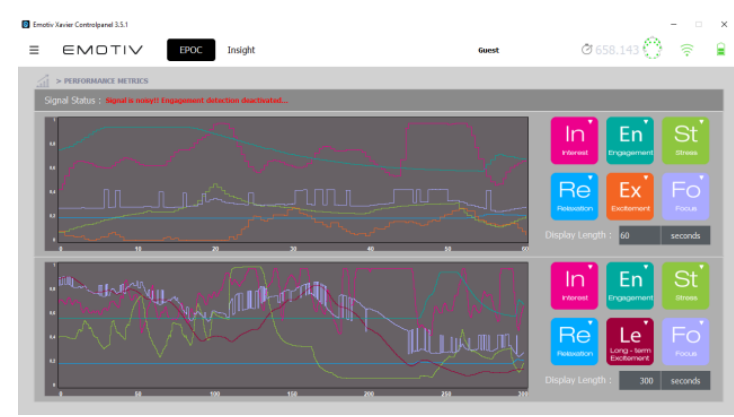

Fig. 3 Software Emotiv Xavier ControlPanel - performance metrics.

The individual performance metrics are color-coded. It is also possible to adjust the length of two time frames. At the top of the picture, we can see the signal status. The signal can be either high quality, reduced or unmeasured. At the top right of the picture, we can check the signal quality of each sensor.

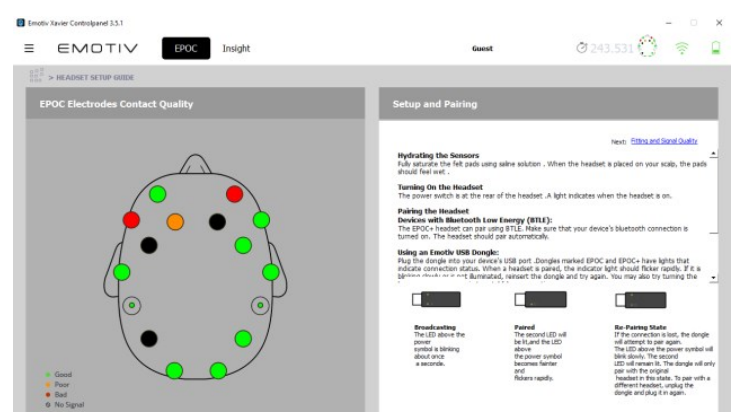

Fig. 4 Software Emotiv Xavier ControlPanel - electrodes contact quality, setup, and pairing.

Here we can see the setup, pairing, and color-coded signal electrodes contact quality. In the upper part, we can also see which device is currently connected and how long it took to setup the device. 


\section{Experimental part}

\subsection{Visual stimuli}

The presentation was made containing ten images where each is displayed after six seconds. Length of the overall record is one minute. Various types of images have been selected. The presentation contains a sequence of pictures of cute animals, funny dog, hedgehog in a mug, starved kids, love, cute bunny, scary house, sea, spider, and nature in the spring.

\subsection{Auditory stimuli}

In this section, six different types of sounds and music were edited and modified. Each lasting twenty seconds. Length of the overall record is two minutes. It contains relaxing music, funny music, death metal, fairy tale, pop music, and horror sounds.

\subsection{Measurement using Emotiv Epoc+}

The first step is to charge the headset. The second step is to hydrate the sensors. Sensors must be very well hydrated to ensure good contact quality. The third step is to install the sensors. The fourth step is to turn on the headset. For connection USB cable is used here. The last step is fitting the headset with good contact quality.

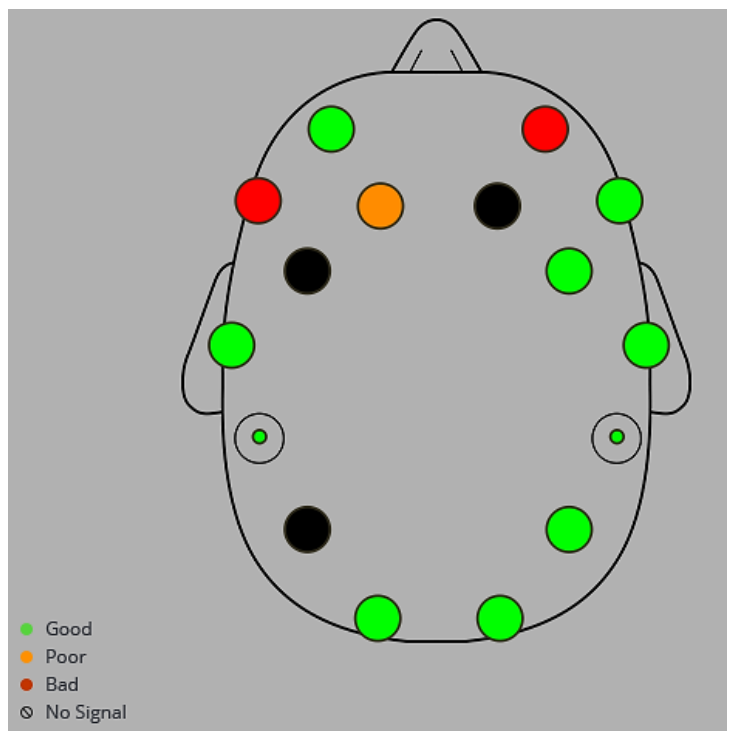

Fig. 5 Epoc +- bad electrodes contact quality.

If we cannot achieve good signal quality for all sensors, we can additionally wet them again.

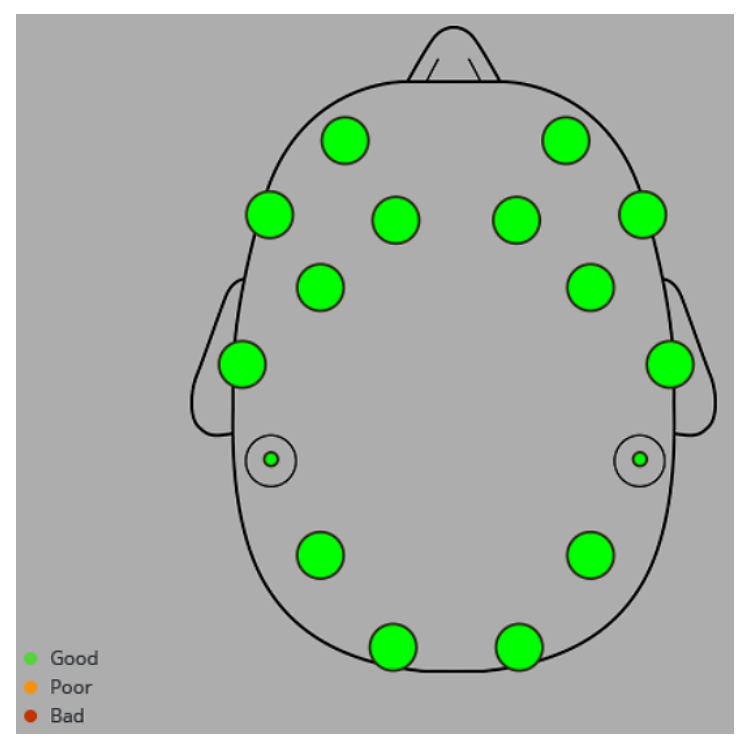

Fig. 6 Epoc +- good electrodes contact quality.

\subsubsection{Visual stimuli - subject 1}

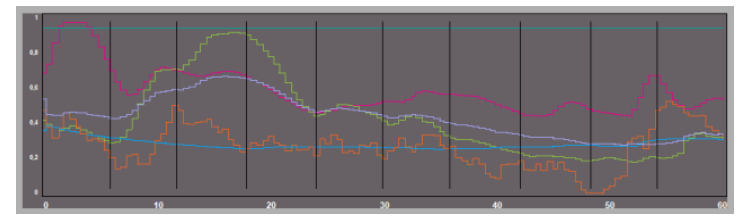

Fig. 7 Subject 1 - brain signals measured by Epoc + headset responding to visual stimuli.

We can see reactions to various visual stimuli on the graph. A significant increase in interest was observed in the picture of cute animals, funny dog, and spider. The stress and focus graphs leveled up in the picture of a funny dog and hedgehog in a mug. Relaxation was almost at the same level during the measurement. A huge increase in excitement can be seen, for instance, in the picture of a funny dog and spider.

\subsubsection{Visual stimuli - subject 2}

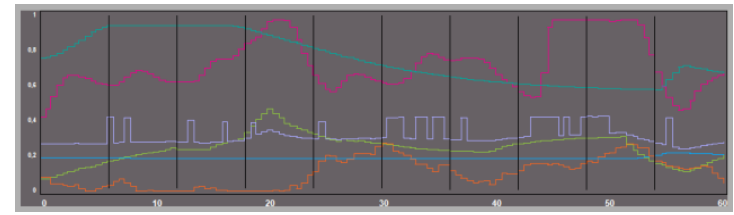

Fig. 8 Subject 2 - brain signals measured by Epoc + headset responding to visual stimuli.

Major changes in the graph were observed on each individual transitions between images. For example, we 
can see a huge increase in interest in the picture of starved kids and the picture of the sea. Increased engagement can be seen in the picture of a funny dog and a picture of nature in the spring. We can see a level up in stress while watching images of starved kids and a scary house. Relaxation slightly increased in the last picture of nature in spring. A raise can be seen in the excitement graph while watching the picture of love, cute bunny, sea, and spider. Each time we can see an increment in focus signal on significant parts of pictures between different images.

\subsubsection{Auditory stimuli - subject 1}

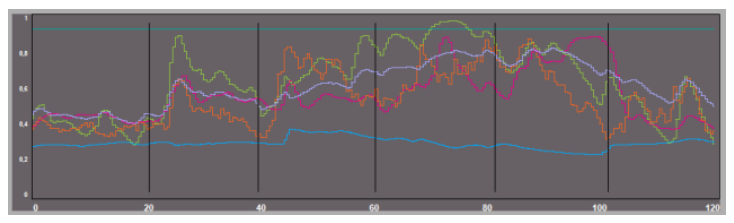

Fig. 9 Subject 1 - brain signals measured by Epoc + headset responding to auditory stimuli.

The graph shows significant changes in individual auditory stimuli. For example, the interest increased the most while listening to the funny music, jingle for fairy tale and in pop music. The level of stress increased the most in the funny music, death metal and horror sounds. Relaxation slightly increased in the death metal. This may be due to the fact, that the subject likes death metal. The level of excitement changed depending on the different genres. The biggest jump in excitement was while listening to death metal. We can also see that the focus was increased while listening to funny music and horror sounds.

\subsubsection{Auditory stimuli - subject 2}

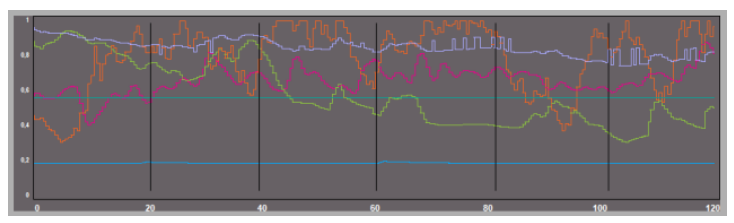

Fig. 10 Subject 2 - brain signals measured by Epoc + headset responding to auditory stimuli.

From the graph, we can see that the interest and excitement increased individually according to the popular genre of the subject. The stress level also gradually decreased and increased according to the type of music. For example, stress has increased the most while listening to the horror sounds. Relaxation slightly increased while listening to funny music and the fairy tale. We can also see that the focus is increased significantly while changes in tonality and music.
We may notice that some signals may not change at all if the sensor does not have a good signal.

\section{Discussion}

During the last decades, a new technology BCI has emerged by which the human brain can directly communicate with the environment. BCIs offer a way of bypassing typical nerve pathways by providing novel output pathways to interact with a variety of applications. Though research into BCI technology is still in early phases, many significant developments have been made. [3] Research in BCIs has achieved impressive progress towards implementing assistive technologies for restoration or substitution of lost motor capabilities, as well as supporting technologies for healthy people. However, effective implementation of these interfaces from proof-of-concept prototypes into reliable real-time applications is still in progress. In one study was shown, that the performance of the Emotiv Epoc+ headset is comparable to that found in professional devices. [6]

Another study evaluated the detection accuracy of Emotiv Epoc+ intended for personal use by normal, healthy users. Results found the system to perform significantly better than a chance for all mental actions, and improve over time with additional training data. These results indicate the Emotiv Epoc+ system performs its function as a BCI with an acceptable level of accuracy, yielding many new possibilities. [7] Low-cost EEG headset devices are fast becoming a key instrument for $\mathrm{BCI}$ applications. However, there are some problems with using EEG devices like BCI illiteracy and artifacts. [8]

The noninvasive recording procedure is safe and easy to apply. An Emotiv Epoc+ headset is a relatively cheap and easily available tool for research, games, etc.

\section{Conclusion}

This study investigated the usability BCI of Emotiv Epoc + headset for communication. The experimental evaluation of the device was performed with two participants. Overall, it was processed by four measurements. To examine brain activity the record of brain activity from Epoc + device responding to visual and auditory stimuli was used. One subject was a man with short hair. Setup time was about five minutes. The second subject was a woman with long and dense hair. The helmet setup was about nine minutes. The values of individual subjects to various stimuli are very different, possibly due to differences between the participants. Each sensor in headset measures different performance metrics. Thus, the failure of a sensor can cause a loss of performance metrics to which it belongs.

In conclusion, the applicability of the Epoc+ helmet is sufficient for both scientific and other research purposes. 
This work was supported by IGA (Internal Grant Agency) of Tomas Bata University in Zlin under the project No. IGA/FAI/2019/005.

\section{References}

1. G. Schalk. In Ch.S. Nam, A. Nijholt, F. Lotte, Braincomputer interfaces handbook: technological and theoretical advances, Boca Raton: Tayl. \& Franc., CRC Press (2018)

2. Ch.S. Nam, A. Nijholt, F. Lotte, Brain-computer interfaces handbook: technological and theoretical advances, Boca Raton: Tayl. \& Franc., CRC Press (2018)

3. Ch.S. Nam, I. Choi, A. Wadeson, M. Whang. In Ch.S. Nam, A. Nijholt, F. Lotte, Brain-computer interfaces handbook: technological and theoretical advances, Boca Raton: Tayl. \& Franc., CRC Press (2018)

4. S. Das, D. Tripathy, J.L. Raheja, Spring. Br. in App. Sci. and Tech. (2019)

5. Emotiv: Emotiv Epoc+ - 14 Channel Wireless EEG Headset [online]. Available on: https://www.emotiv. com/epoc/ (2019)

6. J.-A. Martinez-Leon, J.-M. Cano-Izquierdo, J. Ibarrola, Exp. Sys. with App. 49 (2016)

7. G.S. Taylor, Ch. Schmidt, Proc. of the Hum. Fact. and Erg. Soc. Ann. Meet. 56(1) (2016)

8. R. Maskeliunas, R. Damasevicius, I. Martisius, M. Vasiljevas, PeerJ 4:e1746 (2016) 